\title{
Breast cancer care in northern Ethiopia - cross-sectional analysis
}

\author{
Biniyam Tefera Deressa ${ }^{1,2^{*}}$ D, Nikola Cihoric ${ }^{2}$, Eugenia Vlaskou Badra ${ }^{2},{\text { Alexandros } \text { Tsikkinis }^{2} \text { and Daniel Rauch }}^{3,4}$
}

\begin{abstract}
Background: In Ethiopia, the incidence of new cases of breast cancer is currently increasing resulting to high rates of morbidity and mortality. Breast cancer is by far the most common cancer accounting for more than one out of three cancer cases in women and one out of every five in the general population. The study was conducted in University of Gondar Hospital cancer center, located in the North-West Ethiopia; to evaluate the clino-pathologic characteristics of breast cancer and care provided for patients.

Methods: All biopsy proven breast cancer patients treated between 2016 and 2017, were identified and information regarding histology, stage, therapeutic procedure and follow up was retrospectively collected from their individual medical records and descriptive analysis was done.

Results: Among 82 patients treated, 67 (82\%) were women and 15 (18\%) were men. The median age at the time of diagnosis was 45 years (25-82 years). Operation was performed for 56 (68\%) patients. The predominant histology was ductal carcinoma in 61 patients (74\%), followed by breast carcinoma of No Special Type (NST) in 17 (21\%). The late presentation of the patients and the advanced stage at the time of presentation was observed in most of the patients. Chemotherapy was administered in 79 (96\%) patients. Radiotherapy was not available in the hospital.
\end{abstract}

Conclusion: Breast cancer incidence is rising and becoming a major public health problem in Northern Ethiopia. Breast cancer care in northern-Ethiopia is limited in terms of both pathology, imaging and the offered treatment modalities, which need to be improved.

Keywords: Breast cancer, University of Gondar Hospital, Northern Ethiopia

\section{Background}

Breast cancer is the most frequently occurring cancer in women, with approximately 1.7 million new cases and nearly 522,000 related deaths worldwide in 2012 [1]. In Ethiopia the incidence of new cases of breast cancer is currently increasing and has become one of the most common cancer types causing high rates of morbidity and mortality [2-4]. According to the study by Memirie et al. breast cancer is the most common cancer, constituting $33 \%$ of the cancers in women and $23 \%$ of all cancers in Ethiopia. The Estimated national Age Standardized Incidence rate for women was about 43 per 100,000 population [5].

\footnotetext{
* Correspondence: biniyamtefera.md@gmail.com

${ }^{1}$ University of Gondar, Gondar, Ethiopia

${ }^{2}$ Department of Radiation Oncology, Inselspital, Bern University Hospital,

University of Bern, Bern, Switzerland

Full list of author information is available at the end of the article
}

The recently reported, first data from the Addis Ababa City Population based cancer registry shows that breast cancer is the most common cancer in women and one of the top Ten cancers in men. Breast cancer incidence has shown a steady yearly growth at the Tikur Anbessa Specialized Hospital, the only hospital in the country where cancer patients have access to oncology care $[6$, 7]. Furthermore, the majority of patients have at the time of presentation advanced disease with $67 \%$ presented with locally advanced and $25 \%$ metastatic disease [8]. The combination of all the aforementioned factors leads to unsatisfactory treatment outcomes $[8,9]$.

The Ethiopian government has recognized this as a major problem and has set a priority the advancement of cancer care in the country. In 2015 the Ethiopian healthcare authorities developed a cancer control plan, which is focusing on the prevention as well as the general improvement of cancer care in the country $[10,11]$. 
One of the most significant steps taken was the establishment of 6 specialized cancer centers at the teaching university hospitals of Gondar, Hawassa, Mekelle, Jimma, Haramaya and St. Paul's Hospital [12].

\section{Cancer care at the university hospital of Gondar}

The University of Gondar Hospital is a tertiary teaching hospital, located in the North-West Ethiopia providing healthcare for a population of approximately 5,000,000 people. The hospital has around 600 beds with 400,000 patients treated annually. The hospital has more than sixty years' experience in teaching, research, health care delivery and other community services employing 250 general practitioners, 20 internal medicine specialists, 14 surgeons, 12 pediatricians, 7 gynecologists and more than 30 other medical specialists. The University Hospital also has more than Ten years of experience and participation in clinical trials focusing mainly on infectious diseases such as leishmania and HIV infection [13, 14]. Until recently however, specialized and comprehensive cancer care was not offered at the hospital, with just selected cases being operated on and with the bulk of diagnostic and interventional procedures being referred to the Tikur Anbessa Specialized Hospital located 900 $\mathrm{km}$ from Gondar.

In January 2015 the Gondar University Hospital together with the BEZA Association of Switzerland, established the first cancer treatment center in the region [15]. The center includes Ten beds for outpatients as well as an in-patient care unit with Eight trained nurses, Two general practitioners and One senior oncology resident from the Tikur Anbessa Hospital on a monthly rotation. Furthermore, a senior oncologist from Switzerland regularly visits the center. One of the major achievement of the center was the establishment of a regular multidisciplinary tumor board consisting of a group of medical professionals including a pathologist, a radiologist, a surgeon and the attending physician.

The histopathological evaluation is limited on the morphology, with immunohistochemistry and other molecular tests currently unavailable. The department of radiology is equipped with a CT-Scanner, an X-ray machine and Ultrasound devices. The University of Gondar Hospital cancer center provides medical oncology service such as the administration of chemotherapy and hormone therapy for several tumor entities. Radiotherapy is still not available at the center and those patients requiring radiotherapy are referred to Tikur Anbessa Hospital in Addis Ababa.

Breast cancer is one of the most common cancer entities treated at the University of Gondar Hospital, therefore a retrospective evaluation was conducted to detect patterns of care and evaluate early results [16].

\section{Methods}

The medical record review was done by physicians working in the center. First, we have retrieved medical records of all breast cancer patients. Secondly, we have reviewed records and identify variables that can be extracted from the record. The completeness of the record was ascertained by the primary investigator. Data was collected on tumor stage, lymph node status, and presence of metastasis. The staging in Gondar University Hospital is based on the presence or absence of symptoms and clinical evidence of the disease. In addition to the clinical evaluation, every patient with locally advanced disease was evaluated at least with a chest $\mathrm{x}$-ray and abdominal ultrasound. Patients were staged according to the TNM staging system of the American Joint Committee on Cancer (AJCC), seventh edition [17]. The date of morphological confirmation of breast cancer was considered as date of diagnosis. Pretreatment evaluation included evaluation of complete blood count, renal function tests and liver function tests. In addition to staging data, we have collected data on therapeutic procedures and follow-up. The study was conducted according to the local ethical guidelines.

\section{Result}

From December 2016 to November 2017, 82 patient were treated at the center. Sixty-seven patients (n:67, $82 \%)$ were females and 15 male (n:15, 18\%). The median age for all patients was 45 years (range from 25 to 82 years). About $47 \%$ of the patients were 40 years old or less, $27 \%$ between 40 to 50 years of age and the rest $26 \%$ were above age 50 . Of the 67 female patients 38 patients (57\%) were premenopausal and 29 patients (43\%) were post-menopausal. The 15 male patients were generally older than their women counterparts with a median age of 65 (range 40 to 82 years). The age distribution is depicted in Table 1.

The main presenting symptoms at presentation was a longstanding breast mass in 65 patients $(79 \%)$ and breast ulceration in 15 patients (18\%). Only one patient reported breast pain as initial symptom and one patient presented with pain outside of the breast. In 48 patients (59\%) the lesion was in the right breast, in 32 patients (39\%) in the left breast and 2 patients $(2 \%)$ had bilateral breast lesions. The median duration from first symptom to the time of diagnosis was twelve months (range 3 weeks to 5 year) (Table 1 ).

All of the patients had a metastatic work up at least with Chest X-Ray and abdominal Ultrasound. One of the 82 patients had a mammography as initial work-up and $23(28 \%)$ of the patients had a breast ultrasound.

Fifty-six (68\%) patients received an operation, the most common surgical procedure was modified radical mastectomy $(n=51)$, followed by simple mastectomy $(n=2)$ 
Table 1 Clinical and pathological characteristics of the patients

\begin{tabular}{|c|c|c|}
\hline Characteristics & Number & Proportion \\
\hline Total Population & 82 & $100 \%$ \\
\hline \multicolumn{3}{|l|}{ Duration of symptoms in months } \\
\hline$\leq 4$ & 8 & $10 \%$ \\
\hline $5-8$ & 20 & $24 \%$ \\
\hline $9-16$ & 28 & $34 \%$ \\
\hline $17-24$ & 15 & $18 \%$ \\
\hline$\geq 25$ & 11 & $13 \%$ \\
\hline \multicolumn{3}{|l|}{ Menopausal Status (Women Only) } \\
\hline Pre-menopausal & 38 & $57 \%$ \\
\hline Post-menopausal & 29 & $43 \%$ \\
\hline \multicolumn{3}{|l|}{ Main presenting Symptom } \\
\hline Breast pain & 1 & $1 \%$ \\
\hline Breast Swelling & 65 & $79 \%$ \\
\hline Pain outside breast & 1 & $1 \%$ \\
\hline Ulceration & 15 & $18 \%$ \\
\hline \multicolumn{3}{|l|}{ Lateralization } \\
\hline Right Breast & 48 & $59 \%$ \\
\hline Left Breast & 32 & $39 \%$ \\
\hline Bilateral & 2 & $2 \%$ \\
\hline \multicolumn{3}{|l|}{ Type of Surgery performed } \\
\hline Surgery was not performed & 26 & $32 \%$ \\
\hline Modified Radical Mastectomy & 51 & $62 \%$ \\
\hline Simple Mastectomy & 2 & $2 \%$ \\
\hline Palliative Mastectomy & 3 & $4 \%$ \\
\hline \multicolumn{3}{|l|}{ Histology } \\
\hline Ductal Carcinoma & 61 & $74 \%$ \\
\hline Breast Cancer NST & 17 & $21 \%$ \\
\hline DCIS & 1 & $1 \%$ \\
\hline Lobular Carcinoma & 1 & $1 \%$ \\
\hline Mucinous Carcinoma & 1 & $1 \%$ \\
\hline Papillary Carcinoma & 1 & $1 \%$ \\
\hline \multicolumn{3}{|l|}{ Grade of Differentiation } \\
\hline G-1 & 14 & $17 \%$ \\
\hline $\mathrm{G}-2$ & 30 & $37 \%$ \\
\hline G-3 & 26 & $32 \%$ \\
\hline Not reported & 12 & $15 \%$ \\
\hline \multicolumn{3}{|l|}{ First-line chemotherapy Given } \\
\hline$A C$ & 50 & $61 \%$ \\
\hline FAC & 14 & $17 \%$ \\
\hline$A C+$ taxol & 13 & $16 \%$ \\
\hline Chemotherapy not started & 3 & $4 \%$ \\
\hline CAF & 1 & $1 \%$ \\
\hline CMF & 1 & $1 \%$ \\
\hline
\end{tabular}

Table 1 Clinical and pathological characteristics of the patients (Continued)

\begin{tabular}{lll}
\hline Characteristics & Number & Proportion \\
\hline Hormonal Therapy & & \\
Anastrazole & 6 & $7 \%$ \\
No hormonal therapy & 22 & $27 \%$ \\
On Chemotherapy & 36 & $44 \%$ \\
Tamoxifen & 18 & $22 \%$ \\
\hline
\end{tabular}

and palliative mastectomy $(n=3)$. Surgery was omitted in $26(32 \%)$ patients due to the advanced stage of the disease with metastasis at presentation (Table 1)

The predominant histology was ductal carcinoma in 61 patients $(74 \%)$, followed by breast carcinoma of No Special Type (NST) in 17 (21\%) patients. Ductal Carcinoma In-Situ (DCIS), lobular carcinoma, mucinous carcinoma and papillary carcinoma were found in one patients each. The grade of differentiation was well differentiated in $14(17 \%)$, moderately differentiated in 30 (37\%), poorly differentiated in $26(32 \%)$ and unknown in 12 (15\%) patients. Surgical margin status was reported for 29 patients, with 23 patients having surgical margins free of tumor and 6 infiltrated by the tumor. Since the immunohistochemistry tests are not fully installed in the hospital, only 2 patients had tests for hormonal status (expression of estrogen and progesterone receptors) to which both were found to be positive (Table 1).

The stage at presentation was locally advanced or metastatic for most of the patients. Distribution according to stage at presentation is plotted on Table 1.

All but three patients (96\%) received chemotherapy. Of those 50 patients (61\%) received Adriamycin and Cyclophosphamide (AC-regimen), 14 patients (17\%) Fluorouracil, Adriamycin and Cyclophosphamide (FAC-regimen), and 13 patients (16\%) Adriamycin and Cyclophosphamide plus Paclitaxel (AC-Taxol regimen). Cyclophosphamide, Methotrexate and Fluorouracil (CMF-regimen) and Fluorouracil, Adriamycin and Cyclophosphamide (FAC-regimen) were each administered in one patient each. Among the patients who completed a full course of chemotherapy, 24 patients received empiric hormonal therapy and 22 patients did not receive any hormonal treatment. The rest of the patients were on chemotherapy treatment. The summary of the clinical and pathologic characteristics of patients treated in University of Gondar hospital cancer center is shown in Table 1.

\section{Discussion}

Most of the patients treated at the Gondar university hospital cancer center were young with almost half of them being less than 40 years old. This finding is similar to the reports of previous breast cancer studies completed in Ethiopia [7, 9, 18-20]. This however, is 
different from the current trend in western countries where only less than $7 \%$ of the patients diagnosed are younger than 40 years of age $[21,22]$. The reason for the high number of younger patients in Ethiopia is currently unclear. A potential explanation for this is possibly the demographics of Ethiopia and the high percentage of younger individuals in the population of the country [18, 23]. Indeed, this is a logical explanation since reports in our findings and other previous studies were not age adjusted. Nevertheless, several studies have shown that tumors arising in younger patients are more aggressive and those patients are at a higher risk of relapse and death not only due to their age but also due to the unique tumor biology [24-26]. Therefore, further research is required to identify or exclude potential underlying genetic or environmental factors that would render women in Ethiopia more prone to develop the disease at a young age.

Another finding of our study is the high proportion of male breast cancer, which accounts for $18 \%$ of the total patient population during a calendar year. Unlike breast cancer in women, the median age of breast cancer in men in our findings is similar to the median age reported in western countries [27]. A similar percentage of male breast cancer patients was reported from this hospital in 2016, with breast cancer being among the top 10 cancers diagnosed in men [16]. The rarity of the disease worldwide which accounts for less than $1 \%$ makes this finding interesting [27]. The reason/s for the higher incidence of male breast cancer is not clear. A possible explanation for this are unique cultural factors and/or economic reasons that would explain why men seek healthcare in rural Ethiopia more often than women do [28]. However, reports from the Addis Ababa city population based cancer registry, which includes mostly urban population shows a higher proportion of male breast cancer cases, of about $7 \%$ and still one of the top 10 cancers in men [2]. In conclusion, other possible environmental or genetic factors might be implicated in the high incidence of male breast cancer in Ethiopia. Considering the fact that the higher percentage of male breast cancers is encountered in other Sub-Saharan countries as well as blacks living in the United States where the higher male-to-female ratio is also seen, it is possible that a yet unknown genetic component is potentially instrumental for this phenomenon [29]. Since male breast cancer biology or treatment is not well understood due to its rarity, Ethiopia could be one of the study sites for future clinical trials to boost our knowledge on the topic.

The other findings of our study is the late presentation of the patients and the advanced stage at the time of presentation. About $85 \%$ of the cases were diagnosed at a stage III and IV. This finding was similar with previous breast cancer studies from Ethiopia and other African countries such as Tanzania, Uganda and Egypt [7, 9, 3032]. The absence of screening combined with the low rate of cancer awareness and unavailability of multi-modality treatment may be the reason for the late presentation and diagnosis at an advanced disease stage. It is obvious that the late stage at presentation negatively affects the breast cancer survival [33]. As it was clearly shown on the recent publication of Weiner et al., the average survival probability of metastatic breast cancer in Ethiopia was about 12 months which was significantly lower than western countries [34]. Together with lack of standard therapy, late stage at presentation could be one of the reasons for the higher mortality rates attributed to breast cancer patients [34]. Although the incidence rate of breast cancer is lower in Africa than in high income countries, there is a disproportionately higher number of mortality $[35,36]$. The age-standardized breast cancer incidence rate is 70.08 in Europe and 28.66 in Africa, while the mortality rate is about 15.93 in Europe and 13.72 in Africa [37, 38]. The five-year survival rates is around $80 \%$ in the high-income countries and $40 \%$ in the low-income countries [39]. The higher mortality rates for breast cancer in Africa and other LMICs, is due to the rising incidence as well as late-stage diagnoses and limited access to treatment [40].

The major limitation of the present study is its small sample size. The total number of patients treated in the center could be relatively smaller compared to the total population in the area. This could be due to lack of awareness of the population, seeking of alternative traditional treatments or limitation of knowledge and diagnostics facilities from health care providers. The other reasons could be due to lack of information and thus, less referral of the diagnosed or surgically treated patients to the center as it was newly established.

We believe that the strength of the study could be increased if it had been possible to show the outcome of the breast cancer patients in the hospital in terms of overall survival or progression free survival. Our reason for not including survival analysis was that, the medical record of patients existing during the study period didn't have detailed address or phone number of the patients which makes it difficult to get vital status. In spite of these limitations, our first report on breast cancer clinical, pathologic and treatment pattern may have an impact in sensitizing third parties and thus ultimately improving the healthcare offered. It could also be the base for future larger studies.

\section{Conclusion}

Breast cancer incidence is rising and becoming a major public health problem in Northern Ethiopia. Breast cancer care in northern-Ethiopia is limited in terms of both 
pathology, imaging and the offered treatment modalities, which need to be improved. Furthermore, Ethiopia could potentially become one of the major research areas in terms of younger individuals and men with diagnosed breast cancer.

\section{Abbreviations}

AC-regimen: Adriamycin and Cyclophosphamide; AC-Taxol

regimen: Adriamycin and Cyclophosphamide plus Paclitaxel; AJCC: American Joint Committee on Cancer; CMF-regimen: Cyclophosphamide, Methotrexate and Fluorouracil; DCIS: Ductal Carcinoma In-Situ; FAC-regimen: Fluorouracil, Adriamycin and Cyclophosphamide; HIV: Human Immuno-deficiency Virus; LMICs: Low- and Middle-Income Countries; NST: No Special Type;

TNM: Tumor, Node, Metastasis

\section{Acknowledgements}

The Doctors and Nurses working in the University of Gondar Cancer Center

\section{Funding}

The authors did not receive any funding from other bodies.

\section{Availability of data and materials}

The data that support the findings of this study are available from University of Gondar cancer center, but restrictions apply to the availability of these data, which were used under license for the current study, and so are not publicly available. Data are however available from the authors upon reasonable request and with permission of University of Gondar cancer center.

\section{Authors' contributions}

BTD: conception and design of study, acquisition, collection and assembly of data, drafting the manuscript and final approval. NC: analysis and interpretation of data. EVB: Final approval. AT: Revising for important intellectual content of the manuscript. DR: Conception, design, and Final approval. All authors read and approved the final manuscript.

\section{Ethics approval and consent to participate}

The study was conducted according to the University of Gondar ethical guidelines and approval was received from the University of Gondar ethical committee.

The study was conducted without individual informed consent, as the study relied on retrospective data collected as part of routine patient care and the data was anonymous.

\section{Consent for publication}

Not applicable.

\section{Competing interests}

The authors declare that they have no competing interests.

\section{Publisher's Note}

Springer Nature remains neutral with regard to jurisdictional claims in published maps and institutional affiliations.

\footnotetext{
Author details

${ }^{1}$ University of Gondar, Gondar, Ethiopia. '2Department of Radiation Oncology, Inselspital, Bern University Hospital, University of Bern, Bern, Switzerland. ${ }^{3}$ Regional Hospital Thun, Thun, Switzerland. ${ }^{4}$ Department of Medical Oncology, Inselspital, Bern University Hospital, University of Bern, Bern, Switzerland.
}

Received: 6 September 2018 Accepted: 12 April 2019 Published online: 25 April 2019

\section{References}

1. Torre LA, et al. Global cancer statistics, 2012. CA Cancer J Clin. 2015:65(2): $87-108$

2. Timotewos $\mathrm{G}$, et al. First data from a population based cancer registry in Ethiopia. Cancer Epidemiol. 2018;53:93-8.
3. Lukong KE, Ogunbolude Y, Kamdem JP. Breast cancer in Africa: prevalence, treatment options, herbal medicines, and socioeconomic determinants. Breast Cancer Res Treat. 2017;166(2):351-65.

4. Abate $Y$, et al. Trends of breast cancer in Ethiopia. Int J Cancer Res Mol Mech. 2016;2:1-5

5. Memirie ST, et al. Estimates of Cancer incidence in Ethiopia in 2015 using population-based registry data. J Glob Oncol. 2018:4:1-11.

6. Woldeamanuel YW, Girma B, Teklu AM. Cancer in Ethiopia. The Lancet Oncology. 2013;14(4):289-90

7. Kantelhardt EJ, et al. Cervical cancer in Ethiopia: survival of 1,059 patients who received oncologic therapy. Oncologist. 2014;19(7):727-34.

8. Tigeneh W, et al. Pattern of cancer in Tikur Anbessa specialized hospital oncology center in Ethiopia from 1998 to 2010. Int J Cancer Res Mol Mech. 2015;1(1).

9. Ersumo T. Breast cancer in an Ethiopian population, Addis Ababa. East and Central African Journal of Surgery. 2006;11(1):81-6

10. pinkredribbon. Official launch of the Ethiopian National Cancer Control Plan. Pink ribbon/red ribbon; 2015

11. FMoH. National Cancer Control Plan, 2016-2020. International Cancer contro partnership (ICCP); 2015.

12. Xuequan M. Ethiopia to set up 5 specialized cancer treatment centers XINHUANET; 2018

13. Diro $\mathrm{E}_{\text {, et }}$ al. Long-term clinical outcomes in visceral Leishmaniasis/human immunodeficiency virus-Coinfected patients during and after Pentamidine secondary prophylaxis in Ethiopia: a single-arm clinical trial. Clin Infect Dis. 2018;66(3):444-51.

14. Olende R. Phase III clinical study in Ethiopia launched to test two treatments for HIV-visceral Leishmaniasis co-infected patients. Drug for Neglected Disease initiative. 2014

15. Deressa BT. A journey of a thousand miles begins with a single step: lessons from University of Gondar Cancer Center, Ethiopia. In: Golbal RT; 2017

16. Tefera B, et al. Patterns of Cancer in University of Gondar Hospital: northWest Ethiopia. J Oncol Med \& Pract. 2016;1(106):2.

17. Edge SB. AJCC cancer staging manual, vol. 7: Springer; 2010. p. 97-100.

18. Hadgu $E$, et al. Breast cancer in Ethiopia: evidence for geographic difference in the distribution of molecular subtypes in Africa. BMC Womens Health. 2018;18(1):40

19. Kantelhardt EJ, et al. The prevalence of estrogen receptor-negative breast cancer in Ethiopia. BMC Cancer. 2014;14:895.

20. Abate S, et al. Trends of breast cancer in Ethiopia. Int J Cancer Res Mol Mech. 2016;2(1)

21. Anders CK, et al. Breast cancer before age 40 years. Semin Oncol. 2009:36(3): 237-49.

22. Fredholm H, et al. Breast cancer in young women: poor survival despite intensive treatment. PLoS One. 2009:4(11):e7695.

23. Akarolo-Anthony SN, Ogundiran TO, Adebamowo CA. Emerging breast cancer epidemic: evidence from Africa. Breast Cancer Res. 2010;12(Suppl 4):S8.

24. Azim HA Jr, Partridge AH. Biology of breast cancer in young women. Breast Cancer Res. 2014;16(4):427.

25. Gnerlich JL, et al. Elevated breast cancer mortality in women younger than age 40 years compared with older women is attributed to poorer survival in early-stage disease. J Am Coll Surg. 2009;208(3):341-7.

26. Azim HA Jr, et al. Elucidating prognosis and biology of breast cancer arising in young women using gene expression profiling. Clin Cancer Res. 2012 18(5):1341-51.

27. Anderson WF, et al. Male breast cancer: a population-based comparison with female breast cancer. J Clin Oncol. 2009:28(2):232-9.

28. Alemayehu M, Meskele M. Health care decision making autonomy of women from rural districts of southern Ethiopia: a community based crosssectional study. Int J Women's Health. 2017;9:213-21.

29. Ndom P, et al. A meta-analysis of male breast cancer in Africa. Breast. 2012; 21(3):237-41.

30. Mbonde $\mathrm{M}$, et al. Expression of oestrogen and progesterone receptors, $\mathrm{Ki}$ 67, p53 and BCL-2 proteins, cathepsin D, urokinase plasminogen activator and urokinase plasminogen activator-receptors in carcinomas of the female breast in an African population. East Afr Med J. 2001:78(7):360-5.

31. Mousa SM, et al. Patterns of seeking medical care among Egyptian breast cancer patients: relationship to late-stage presentation. Breast. 2011;20(6): 555-61

32. Gakwaya A, et al. Cancer of the breast: 5-year survival in a tertiary hospital in Uganda. Br J Cancer. 2008;99(1):63. 
33. Caplan L. Delay in breast cancer: implications for stage at diagnosis and survival. Front Public Health. 2014;2:87.

34. Weiner $\mathrm{CM}$, et al. Characteristics and follow-up of metastatic breast cancer in Ethiopia: a cohort study of 573 women. Breast. 2018;42:23-30.

35. Ferlay J, et al. Estimates of worldwide burden of cancer in 2008: GLOBOCAN 2008. Int J Cancer. 2010;127(12):2893-917.

36. Ferlay J, et al. Cancer incidence and mortality worldwide: sources, methods and major patterns in GLOBOCAN 2012. Int J Cancer. 2015;136(5).

37. Donepudi $\mathrm{M}$, et al. Breast cancer statistics and markers. J Cancer Res Ther. 2014;10(3):506-11.

38. Torre LA, et al. Global Cancer incidence and mortality rates and trends - an update. Cancer Epidemiology Biomarkers \&amp; Prevention. 2016;25(1):1627.

39. Coleman MP, et al. Cancer survival in five continents: a worldwide population-based study (CONCORD). The lancet oncology. 2008:9(8):730-56.

40. Pace LE, Shulman LN. Breast cancer in sub-Saharan Africa: challenges and opportunities to reduce mortality. Oncologist. 2016;21(6):739-44.

Ready to submit your research? Choose BMC and benefit from:

- fast, convenient online submission

- thorough peer review by experienced researchers in your field

- rapid publication on acceptance

- support for research data, including large and complex data types

- gold Open Access which fosters wider collaboration and increased citations

- maximum visibility for your research: over $100 \mathrm{M}$ website views per year

At BMC, research is always in progress.

Learn more biomedcentral.com/submissions 